\title{
Neonatal mortality in Nepal: A Public Health Concern
}

\author{
Hari Krishna Bhattarai \\ MPH stud(James P. Grant School of Public Health, BRAC University) \\ $\operatorname{MPA}(\mathrm{TU})$
}

The term neonate refers to a live born infant from birth to before reaching 28 completed days of age whereas neonatal mortality rate(NMR) refers to the number of live born babies who die in the first 28 days after birth per 1000 live births (WHO, 2009). Neonatal death is a global public health problem mostly concentrated in low and middle income counties. Globally, out of 130 million newborn, 4 million neonates die each year and 450 newborns die every hour and $99 \%$ of them occurs in middle and low income countries (Lawn, Cousens \& Zupan, 2005). A study done by Lawn, Ketende and Cousens(2006) http://ije.oxfordjournals.org/ content/35/3/706.abstract - aff-4found that the leading causes of neonatal death globally were infections (sepsis/ pneumonia, tetanus, and diarrhoea, 35\%), preterm birth (28\%), and asphyxia (23\%).

According to Nepal Demographic and Health Survey (2006), neonatal death is 33 per 1000 live birth which stands $54 \%$ of total under five and $69 \%$ of infant mortality rate. Which means, every hour, 3-4 neonates die. NMR distribution disparities can be seen on the basis of socioeconomic, educational and geographical areas. NMR in rural was higher as compared to urban areas (urban and rural areas have 25 and 40 death per 1,000 live births respectively) where only $13 \%$ people live in urban areas. Mother's education was inversely related to a neonatal risk of dying. To meet the Millennium Development Goal (MDG) four, NMR need to be reduced from existing 33 in 2006 to 17/1000 live birth in 2015. Nepal millennium development progress report (2010) has mentioned that the under-five mortality rate has decreased significantly in recent years and Nepal is on track to meet millennium development goal on child mortality. But, without reducing neonatal mortality rate, it is not easy to decrease child mortality in coming years.

According to WHO (2010), to achieve MDG, twenty three doctors, nurses and midwives per 10,000 population is necessary to deliver essential maternal and child health services. Whereas, Nepal has only seven. This disparity has further increased due to concentration of health workforce in urban areas. Department of Health Service, Department of Health Service, Annual report (2010) explained that $69 \%$ delivery takes place at home without assistance of health worker and $64 \%$ have not received any postnatal care service. Among the neonatal deaths twothird occurs in first week of life and of those who die in the first week, two-thirds die in the first 24 hours of life. (Bell, Storti and Gamache, 2004).

NMR can be reduced by addressing preventable causes of death through scaling up evidence based public health intervention. Priority interventions based on assessment of available evidence for impact on perinatal and neonatal health status include antenatal care, labor and birth care, immediate newborn care, and postpartum care for mother and newborn. Disease control priority project (2007) mentioned that most of the problems of newborn occur due to lack of basic and essential newborn care, such as ensuring proper breathing, temperature control, hygiene and proper feeding. For example, exclusive breastfeeding provides many benefits to the newborn, such as protecting against infections and allergies and promoting mother-baby bonding. Early initiation of breastfeeding and exclusive breastfeeding can decrease neonatal mortality. In developing countries the greatest advantage of breast milk is its anti-infective property (Cesar JA et al. 1999). It has been shown to decrease diarrhea mortality in the first month. Sixteen percent of neonatal deaths could be prevented if all infants were breastfed from day 1 , and $22 \%$ saved if breastfeeding started within the first hour (Edmond KM et al. 2006). Recent evidence has shown that $41 \%$ decrease in the newborn mortality rate where birth attendants washed their hands before delivery and mothers washed theirs before handling the babies. Tetanus toxoid accounts for about $3 \%$ of neonatal mortality in the developing world. Tetanus toxoid immunization during pregnancy is a simple method to protect and prevent tetanus in the newborn. Bateman (2010) argued saving newborn lives does not require highly specialized staff or equipment. A number of community-based studies have identified and provided evidences of reduced neonatal death focusing on care at home, especially feeding, warming, and cleanliness. A clustered randomized controlled trail study in Uttarpradesh, India by Kumar et al. (2008) showed that focusing to high risk newborn care practice which is socially contextualized and community based, can lead a substantial behavioral modification and reduction of neonatal mortality. Study by Khanal, Zhang \& Khanal (2009) in Morang innovative neonatal intervention program concluded that appropriately trained community level health worker can diagnose and manage neonatal illness. The intervention showed the reduced neonatal fatality.

In Nepal, Community Based Neonatal Care program (CB$\mathrm{NCP}$ ) is being implemenmted through Government and different bilateral and multilateral partners. This intervention is a comprehnsive package including health facility and commununity based care and services. As there are different stragies in the program there should be analysis of the 
effectiveness of the program at all levels i.e. input output and outcome and impact level. At the mean time, To implement develop and implement an effective program to achieve required results is not possible through the implementation of fix strategy only. This should be tailored made (based on the scientifice evidence, cultural, social, life style, geography, educational and economic status) rather that one size fits all.

\section{References}

1. Bateman, M. (2010). Nepal: Saving Newborn Lives to Meet Millennium Development Goal 4.

2. Bell,R., Storti, C. \& Gamache, N. (2004). Care of the newborn reference manual. Save the Children Federation.

3. Cesar JA et al. (1999). Impact of breastfeeding on admission for pneumonia during post neonatal period in Brazil: nested case-control study. British Medical Journal 318:1316-1320

4. Department of health services,Nepal (2010). annual report 2008

5. Disease control priority project. (2007). A Preventable Tragedy: Maternal and Newborn Deaths in West Africa.

6. Edmond KM et al. (2006). Delayed breastfeeding initiation increases risk of neonatal mortality. Pediatrics 117:380-386.
7. Khanal,S., Zhang, W. \& Khanal,S.(2009). The Efficacy of Community Based Intervention in Newborn Care Practices and Neonatal Illness Management in Morang District of Nepal. Life Science Journal 6 (4), 34-40

8. Kumar, V., Mohanty, S., Kumar, A. , Misra, R.P., Santosham, M., Awasthi, S., and et all.(2008). Effect of community-based behaviour change management on neonatal mortality in Shivgarh, Uttar Pradesh, India: a cluster-randomized controlled trial.The Lancet, 272(9644), 1151-1162

9. Lawn, J. E, Cousens, S., \& Zupan, J. (2005). 4 million neonatal deaths: When? Where? Why?The Lancet 365(9462), 891-900.

10. Lawn, J. E, Wilczynska-Ketende, K, Cousens ,S. N.(2006). Estimating the causes of 4 million neonatal deaths in the year 2000. International Journal of Epidemiology. 35 (3),706-718.

11. Ministry of Health and Population, New ERA, and Macro International Inc. (2007) Nepal Demographic and Health Survey 2006. Kathmandu, Nepal: Ministry of Health and Population, New ERA, and Macro International Inc.

12. National Planning Commission (2010).Nepal millennium development progress report 2010.

12. WHO(2010,August). WHO Global Atlas of the Health Workforce.

13. World Health Organization (2009) World Health Statistics Indicator compendium. 